\title{
Power Control for Cognitive Radio Networks Under Channel Uncertainty
}

\author{
Emiliano Dall'Anese, Member, IEEE, Seung-Jun Kim, Member, IEEE, Georgios B. Giannakis, Fellow, IEEE, \\ and Silvano Pupolin, Senior Member, IEEE
}

\begin{abstract}
Cognitive radio (CR) networks can re-use the $\mathrm{RF}$ spectrum licensed to a primary user (PU) network, provided that the interference inflicted to the PUs is carefully controlled. However, due to lack of explicit cooperation between CR and PU systems, it is often difficult for CRs to acquire CR-to-PU channels accurately. In fact, if the $P U$ receivers are off, the sensing algorithms cannot obtain the channels for the PU receivers, although they have to be protected nevertheless. In order to achieve aggressive spectrum re-use even in such challenging scenarios, power control algorithms that take channel uncertainty into account are developed. Both log-normal shadowing and small-scale fading effects are considered through suitable approximations. Accounting for the latter, centralized network utility maximization (NUM) problems are formulated, and their Karush-Kuhn-Tucker points are obtained via sequential geometric programming. For the case where CR-to-CR channels are also uncertain, a novel outage probability-based NUM formulation is proposed, and its solution method developed in a unified fashion. Numerical tests verify the performance merits of the novel design.
\end{abstract}

Index Terms-Cognitive radio, power control, network utility maximization, channel uncertainty, interference modeling, geometric programming.

\section{INTRODUCTION}

C OGNITIVE radio (CR) systems aim to make opportunistic use of the RF spectrum that has been licensed to (but is not fully utilized by) primary user (PU) systems. Provided that the interference channels from the CR transmitters to the PU receivers can be accurately acquired through $\mathrm{CR}$ spectrum sensing, judicious control of transmission powers of the CR systems can protect the PU transmissions while maximizing the CR network performance [1]. Such a coexistence strategy is often termed as spectrum underlay [2].

However, typically due to lack of collaboration mechanisms between PU and CR systems, reliably detecting the presence of PU transmissions, and accurately estimating the CR-to-PU channels require considerable effort. Particularly challenging is to acquire these channels for "passive" PU nodes during the

Manuscript received February 20, 2011; revised June 2, 2011 and August 2, 2011; accepted August 3, 2011. The associate editor coordinating the review of this paper and approving it for publication was M. Ardakani.

This work was supported by NSF grants CCF-0830480, CCF-1016605, ECCS-0824007, and ECCS-1002180; and QNRF grant NPRP 09-341-2-128.

E. Dall'Anese, S.-J. Kim, and G. B. Giannakis are with the Department of Electrical and Computer Engineering, University of Minnesota, 200 Union Street SE, Minneapolis, MN 55455, USA (e-mail: \{emiliano, seungjun, georgios\}@umn.edu).

S. Pupolin is with the Department of Information Engineering (DEI), University of Padova, Via Gradenigo 6/B, 35131, Padova, Italy (e-mail: pupolin@dei.unipd.it).

Digital Object Identifier 10.1109/TWC.2011.081711.110323 time these nodes do not transmit but just listen. Nonetheless, those receivers still need to be protected under the PU-CR hierarchy.

One way to estimate the potential PU receiver locations, and eventually their fading channel gains, is to rely on the idea of channel gain cartography [3]. Once the locations of the PU transmitters are acquired through CR sensing [4], the channel gain maps can predict the corresponding PU coverage regions, in which the PU receivers must reside [5].

$\mathrm{CR}$ power control in underlay scenarios has been studied often under the assumption of perfect CR-to-PU channel knowledge [6]-[8]. Limited-rate feedback from the PU system on instantaneous channel gains was assumed available, and exploited for CR resource allocation in [9]. Focusing on a single CR link and a PU link, [10] analyzed the ergodic capacity under Rayleigh fading using various channel uncertainty models. The present work deals with multiple CR and PU links when both log-normal shadowing and Nakagami small-scale fading are accounted for.

Our approach is to exploit statistical channel knowledge of the CR-to-PU channels, which may be obtained through (imperfect) spectrum sensing, or from the channel gain cartography. Since the wireless channel is often modeled through shadow fading as well as small-scale fading (on top of the deterministic path loss), both sources of uncertainty are taken into account. As PU protection must be enforced strictly, probabilistic constraints are imposed in order to guarantee that the interference power experienced by PU receivers stays below a tolerable level within a prescribed probability.

Since exact evaluation of the interference probabilities is not tractable, approximation techniques are employed. Specifically, the sum of interfering signal powers is approximated as log-normal using the Fenton-Wilkinson method [11]. Then, chance-constrained weighted utility maximization problems are formulated. The problems turn out to be non-convex, but can be reformulated so that their Karush-Kuhn-Tucker (KKT) solutions are obtained via sequential geometric programming (GP). Although KKT solutions capture locally optimal points and do not guarantee global optimality, this is still remarkable considering the fact that the power allocation problems with perfect channel knowledge are also typically non-convex and often tackled using a sequential GP approach [12]. The sequential GP approach can benefit from the availability of efficient interior-point solvers optimized for geometric programs. The optimization is executed at a centralized processor which acquires the necessary channel 
statistics, and feeds back the optimal variables to individual CR nodes through the control channel.

The proposed approach is also extended to the case where the channels between CR transceivers cannot be accurately acquired as well. This may happen due to mobility of the CR nodes, insufficient time for training owing to prolonged PU activity, or ad hoc infrastructure of the CR network. To address such a case, a robust network utility maximization (NUM) problem is formulated, where the signal-to-interference-plusnoise ratios (SINRs) of the CR links are maintained within prescribed outage targets. A log-normal approximation of the SINR statistics proves to be vital for rendering such a formulation tractable.

The rest of the paper is organized as follows. Sec. II describes the system model and formulates relevant optimization problems. Sec. III approximates probabilistic interference constraints. Problem reformulation and solution based on sequential GP are described in Sec. IV. The extension to uncertain CR-to-CR channels is treated in Sec. V. Sec. VI presents the results from numerical tests, and Sec. VII concludes the paper.

\section{System Model And Problem Formulation}

Consider a CR network comprising $K$ transmitter-receiver pairs, sharing spectrum bands licensed to a PU system. In order to make opportunistic use of the spectrum under PUCR hierarchy, the CR system first employs spectrum sensing algorithms to detect the PU system activity in space and time domains [3], [4], and identify relevant parameters including the number of active PUs. Based on the sensing results, power control is subsequently performed to prevent excessive interference to the PU system, and to maximize the CR overall network performance. The focus of the present work is on this resource allocation task.

Let $\mathbf{x}_{k}$ and $\mathbf{u}_{k}$, for $k \in\{1,2, \ldots, K\}$, denote the locations of the $k$-th CR transmitter and receiver, respectively, and $g_{\mathbf{x}_{\mathbf{k}} \rightarrow \mathbf{u}_{\mathbf{k}}}$ the channel gain of link $\mathbf{x}_{k} \rightarrow \mathbf{u}_{k}$, which can be expressed as

$$
g_{\mathbf{x}_{\mathbf{k}} \rightarrow \mathbf{u}_{\mathbf{k}}}=g_{k}|| \mathbf{x}_{k}-\mathbf{u}_{k} \|^{-\eta} s_{\mathbf{x}_{\mathbf{k}} \rightarrow \mathbf{u}_{\mathbf{k}}}\left|h_{\mathbf{x}_{\mathbf{k}} \rightarrow \mathbf{u}_{\mathbf{k}}}\right|^{2}
$$

where $g_{k}$ collects the antenna and other propagation gains, $\eta$ is the path loss exponent, $s_{\mathbf{x}_{\mathbf{k}} \rightarrow \mathbf{u}_{\mathbf{k}}}$ denotes shadow fading, modeled as log-normal, and $h_{\mathbf{x}_{\mathbf{k}} \rightarrow \mathbf{u}_{\mathbf{k}}}$ is the small-scale fading, which is assumed to be Nakagami- $m$ distributed, with $m \geq$ $\frac{1}{2}$ [13, Ch. 2]. It is also assumed that small-scale fading is independent across different links, and of shadow fading. On the other hand, shadowing is allowed to be correlated across links [14], [15].

Let $p_{k}$ denote the transmission power of $\mathrm{CR} k$, and $\mathbf{p}:=$ $\left[p_{1}, \ldots, p_{K}\right]^{T}$ collects the transmit-powers of all $K$ CRs. Also, let $\pi_{k}$ denote the received PU signal power as well as other interference measured at the $k$-th $\mathrm{CR}$ receiver. Then, the instantaneous SINR at CR receiver $k$ can be expressed as

$\gamma_{k}:=\frac{p_{k} g_{\mathbf{x}_{\mathbf{k}} \rightarrow \mathbf{u}_{\mathbf{k}}}}{\sum_{k^{\prime}=1, k^{\prime} \neq k}^{K} p_{k^{\prime}} g_{\mathbf{x}_{\mathbf{k}^{\prime}} \rightarrow \mathbf{u}_{\mathbf{k}}}+\pi_{k}+\sigma_{k}^{2}}, \quad k=1,2, \ldots, K$

where $\sigma_{k}^{2}$ is the receiver noise power at CR $k$. Define $\gamma:=$ $\left[\gamma_{1}, \ldots, \gamma_{K}\right]^{T}$.
Let $\left\{\mathbf{r}_{r}\right\}_{r=1}^{R}$ denote the positions of $R$ PU receivers, and suppose the statistics of the channel gains $\left\{g_{\mathbf{x}_{\mathbf{k}} \rightarrow \mathbf{r}_{\mathbf{r}}}\right\}$ of the CR-to-PU links $\left\{\mathbf{x}_{k} \rightarrow \mathbf{r}_{r}\right\}$ have been acquired. One way to obtain such statistics is to perform spectrum sensing to detect the PUs and estimate the channels to them via reciprocity when also the PU receivers transmit. Alternatively, $\left\{\mathbf{r}_{r}\right\}$ may denote candidate positions of the PU receivers that serve as reference points for constraining interference to the PU system. A way to obtain a reasonable set of reference positions is to rely on the recently introduced notion of RF cartography. For example, channel gain maps were employed as a means to obtain an estimate of the PU coverage region in [5]. In [4], the PU power spectral density (PSD) map was estimated to reveal "crowded" regions in terms of PU presence. Hence, the set $\left\{\mathbf{r}_{r}\right\}_{r=1}^{R}$ may be obtained by discretizing the boundaries of the PU coverage region.

Assuming incoherent superposition of CR waveforms [16], the interference power experienced by PU receiver $r$ due to $\mathrm{CR}$ transmissions is given by

$$
i_{r}:=\sum_{k=1}^{K} p_{k} g_{\mathbf{x}_{\mathbf{k}} \rightarrow \mathbf{r}_{\mathbf{r}}}, \quad r=1,2, \ldots, R .
$$

The power control problem of interest is to maximize the network utility of the CR system while constraining the interference to the PU system. Specifically, upon introducing nonnegative weights $\left\{w_{k}\right\}_{k=1}^{K}$ signifying the link priorities, the following network-wide utility functions are of interest.

i) weighted sum-rate utility: $\mathcal{U}_{1}(\gamma(\mathbf{p})):=\sum_{k=1}^{K} w_{k} \log _{2}\left(1+\gamma_{k}(\mathbf{p})\right)$.

ii) weighted proportional fairness utility: $\mathcal{U}_{2}(\gamma(\mathbf{p})):=\sum_{k=1}^{K} w_{k} \ln \left(\log _{2}\left(1+\gamma_{k}(\mathbf{p})\right)\right)$.

iii) weighted harmonic-mean-rate utility:

$\mathcal{U}_{3}(\gamma(\mathbf{p})):=\left[\sum_{k=1}^{K} \frac{1}{w_{k} \log _{2}\left(1+\gamma_{k}(\mathbf{p})\right)}\right]^{-1}$.

iv) weighted minimum-rate utility: $\mathcal{U}_{4}(\gamma(\mathbf{p})):=\min _{k} w_{k} \log _{2}\left(1+\gamma_{k}(\mathbf{p})\right)$.

Different utility functions correspond to different CR scheduling strategies, and capture the trade-offs between system efficiency and user fairness [17], [18]. System efficiency is maximized by adopting $\mathcal{U}_{1}(\cdot)$, in the sense of weighted total rate of the system is maximized, while it is forgone the most by using $\mathcal{U}_{4}(\cdot)$. On the other hand, in terms of fairness, the order is reversed; i.e., $\mathcal{U}_{4}(\cdot)$ yields the most fair resource allocation, and $\mathcal{U}_{1}(\cdot)$ the least fair one. Utilities $\mathcal{U}_{2}(\cdot)$ and $\mathcal{U}_{3}(\cdot)$ stand in between the two extremes.

If the CR-to-CR channel gains $\left\{g_{\mathbf{x}_{\mathbf{k}} \rightarrow \mathbf{u}_{\mathbf{k}}}\right\}$ as well as the CR-to-PU channel gains $\left\{g_{\mathbf{x}_{\mathbf{k}} \rightarrow \mathbf{r}_{\mathbf{r}}}\right\}$ were perfectly known to the CR network, the optimal transmission power vector $\mathbf{p}$ that maximizes the preceding utilities without causing harmful interference to the PU system would be obtained by solving

$$
\begin{aligned}
(\mathrm{P} 1) \quad \max _{\mathbf{p} \succ \mathbf{0}} & \mathcal{U}_{n}(\gamma(\mathbf{p})) \\
\text { subject to } p_{k} & \leq p_{k}^{\max }, \quad k=1, \ldots, K \\
i_{r}(\mathbf{p}) & \leq i_{r}^{\max }, \quad r=1, \ldots, R
\end{aligned}
$$

where $n \in\{1, \ldots, 4\}, p_{k}^{\max }$ is the maximum allowed transmitpower for CR $k$, and $i_{r}^{\max }$ denotes the maximum interference power that can be tolerated by PU receiver $r$. Problem (P1) is in general non-convex, but it has been reported that a 
successive GP technique can be employed to obtain nearoptimal solution efficiently [12].

In practice, precise knowledge of the CR-to-PU channels requires explicit coordination between the $\mathrm{PU}$ and the $\mathrm{CR}$ systems. In typical CR scenarios where such coordination is infeasible, statistical knowledge of $\left\{g_{\mathbf{x}_{\mathbf{k}} \rightarrow \mathbf{r}_{\mathbf{r}}}\right\}$ may instead be used. In this case, to protect the PU transmissions under channel uncertainty, probabilistic interference constraints are well motivated. Thus, the problem of interest becomes

(P2) $\max _{\mathbf{p} \succ \mathbf{0}} \mathcal{U}_{n}(\gamma(\mathbf{p}))$

$$
\begin{array}{lll}
\text { subject to } & p_{k} \leq p_{k}^{\max }, & k=1, \ldots, K \\
& \operatorname{Pr}\left\{i_{r}>i_{r}^{\max }\right\} \leq \epsilon_{r}, \quad & r=1, \ldots, R
\end{array}
$$

where $\epsilon_{r}>0$ is a prescribed parameter upper-bounding the probability that the CR interference at PU receiver $r$ exceeds a given threshold $i_{r}^{\max }$. On top of the non-convexity issue, an additional challenge in solving (P2) is to obtain a tractable closed-form expression of the probability in $(5 \mathrm{c})$.

In addition to the CR-to-PU channel uncertainty, the channels between the $\mathrm{CR}$ transceivers may also contain uncertainty due, e.g., to insufficient training compared to the speed of channel variation. To address such a case, a chanceconstrained NUM formulation is proposed where outage probability constraints are imposed on a per-CR link basis.

Denote as $\bar{\gamma}_{k}>0$ the SINR target on CR link $\mathbf{x}_{k} \rightarrow \mathbf{u}_{k}$, and $\bar{\gamma}:=\left[\bar{\gamma}_{1}, \ldots, \bar{\gamma}_{K}\right]^{T}$. Also, let $\nu_{k}>0$ be a pre-specified parameter representing an upper-bound to the probability of failing to achieve the SINR target on link $k$, selected according to a link quality requirement. Then, a chance-constrained NUM problem can be formulated as

$$
\max _{\substack{\mathbf{p} \succ 0 \\ \bar{\gamma} \succ 0}} \mathcal{U}_{n}(\bar{\gamma})
$$

subject to $p_{k} \leq p_{k}^{\max }$

$$
k=1, \ldots, K
$$$$
\operatorname{Pr}\left\{i_{r}(\mathbf{p})>i_{r}^{\max }\right\} \leq \epsilon_{r}, \quad r=1, \ldots, R
$$$$
\operatorname{Pr}\left\{\gamma_{k}(\mathbf{p})<\bar{\gamma}_{k}\right\} \leq \nu_{k}, \quad k=1, \ldots, K
$$

where $n \in\{1, \ldots, 4\}$. In section $\mathrm{V}$, an approximation for the SINR will be employed to obtain a tractable constraint in place of (6d). Then, (P3) will be reformulated and solved in the same framework as for solving (P2).

\section{Approximation of Interference Constraints}

In order to tackle (P2), the chance constraints $(5 c)$ must be written explicitly in terms of the optimization variable $\mathbf{p}$. As the random variable (r.v.) $i_{r}$ involves summation of powers affected by possibly correlated shadow fading and small-scale fading, direct characterization of its distribution will not lead to a tractable optimization formulation. To sidestep this hurdle, the distribution of $i_{r}$ is approximated in the sequel.

First, let us focus on the composite fading $l_{\mathbf{x}_{\mathbf{k}} \rightarrow \mathbf{r}_{\mathbf{r}}}:=$ $s_{\mathbf{x}_{\mathbf{k}} \rightarrow \mathbf{r}_{\mathbf{r}}} \cdot\left|h_{\mathbf{x}_{\mathbf{k}} \rightarrow \mathbf{r}_{\mathbf{r}}}\right|^{2}$, which is denoted in $\mathrm{dB}$ as $L_{\mathbf{x}_{\mathbf{k}} \rightarrow \mathbf{r}_{\mathbf{r}}}:=$ $10 \log _{10} l_{\mathbf{x}_{\mathbf{k}} \rightarrow \mathbf{r}_{\mathbf{r}}}$. Upon denoting the mean and the variance of the shadowing component $S_{\mathbf{x}_{\mathbf{k}} \rightarrow \mathbf{r}_{\mathbf{r}}}:=10 \log _{10} s_{\mathbf{x}_{\mathbf{k}} \rightarrow \mathbf{r}_{\mathbf{r}}}$ in dB as $\mu_{S_{\mathbf{x}_{\mathbf{k}} \rightarrow \mathbf{r}_{\mathbf{r}}}}$ and $\sigma_{S_{\mathbf{x}_{\mathbf{k}} \rightarrow \mathbf{r}_{\mathbf{r}}}}^{2}$, respectively, the probability density function (pdf) of r.v. $l_{\mathbf{x}_{\mathbf{k}} \rightarrow \mathbf{r}_{\mathbf{r}}}$ is given by the Gamma-lognormal density

$$
\begin{aligned}
& f_{l_{\mathbf{x}_{\mathbf{k}} \rightarrow \mathbf{r}_{\mathbf{r}}}}(l)=\int_{0}^{\infty}\left(\frac{m}{u}\right)^{m} \frac{l^{m-1}}{\Gamma(m)} e^{-\frac{m l}{u}}
\end{aligned}
$$

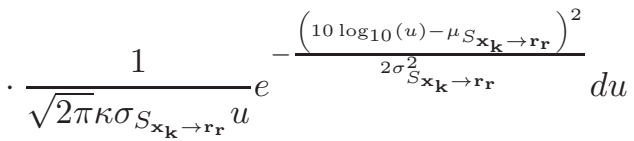

where $\kappa:=\frac{1}{10} \ln 10$, and $\Gamma(\cdot)$ is the Gamma function. It is known that the pdf in (7) can be well-approximated by the log-normal density as [13, Ch. 2]

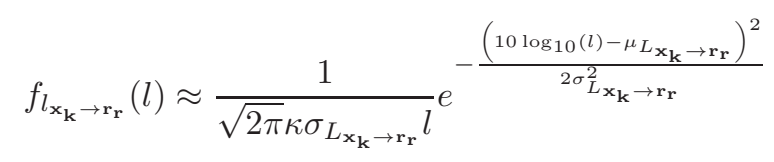

where $\mu_{L_{\mathbf{x}_{\mathbf{k}} \rightarrow \mathbf{r}_{\mathbf{r}}}}$ and $\sigma_{L_{\mathbf{x}_{\mathbf{k}} \rightarrow \mathbf{r}_{\mathbf{r}}}}^{2}$ are expressed in terms of Euler's constant $C \approx 0.5772$ and Hurwitz's zeta function $\zeta(\cdot, \cdot)$ as

$$
\begin{aligned}
& \mu_{L_{\mathbf{x}_{\mathbf{k}} \rightarrow \mathbf{r}_{\mathbf{r}}}}:=\kappa^{-1}\left(-\ln m-C+\sum_{m^{\prime}=1}^{m-1} \frac{1}{m^{\prime}}\right)+\mu_{S_{\mathbf{x}_{\mathbf{k}} \rightarrow \mathbf{r}_{\mathbf{r}}}} \\
& \sigma_{L_{\mathbf{x}_{\mathbf{k}} \rightarrow \mathbf{r}_{\mathbf{r}}}}^{2}:=\kappa^{-2} \zeta(2, m)+\sigma_{S_{\mathbf{x}_{\mathbf{k}} \rightarrow \mathbf{r}_{\mathbf{r}}}}^{2} .
\end{aligned}
$$

The approximation in (8) is quite accurate in the propagation scenarios of practical interest. Specifically, a good approximation is obtained for $\sigma_{S_{\mathbf{x}_{\mathbf{k}} \rightarrow \mathbf{r}_{\mathbf{r}}}} \geq 6 \mathrm{~dB}$ when $m=1$; for $\sigma_{S_{\mathbf{x}_{\mathbf{k}} \rightarrow \mathbf{r}_{\mathbf{r}}}} \geq 4 \mathrm{~dB}$ when $m=2$; and, for any $\sigma_{S_{\mathbf{x}_{\mathbf{k}} \rightarrow \mathbf{r}_{\mathbf{r}}}}$ when $m \geq 4$ [19]. The approximation was used in [16] in a cellular network context, and more recently for interference modeling in CR networks [20].

Under (8), the overall channel gain $g_{\mathbf{x}_{\mathbf{k}} \rightarrow \mathbf{r}_{\mathbf{r}}}$ is also approximately log-normal [cf. (1)]. Let $G_{\mathbf{x}_{\mathbf{k}} \rightarrow \mathbf{r}_{\mathbf{r}}}:=10 \log _{10} g_{\mathbf{x}_{\mathbf{k}} \rightarrow \mathbf{r}_{\mathbf{r}}}$ denote the Gaussian-approximated channel gain in $\mathrm{dB}$ of link $\mathbf{x}_{k} \rightarrow \mathbf{r}_{r}$, and let $\mu_{G_{\mathbf{x}_{\mathbf{k}} \rightarrow \mathbf{r}_{\mathbf{r}}}}:=10 \log _{10}\left(g_{k}\left\|\mathbf{x}_{k}-\mathbf{r}_{r}\right\|^{-\eta}\right)+$ $\mu_{L_{\mathbf{x}_{\mathbf{k}} \rightarrow \mathbf{r}_{\mathbf{r}}}}$ and $\sigma_{G_{\mathbf{x}_{\mathbf{k}} \rightarrow \mathbf{r}_{\mathbf{r}}}}:=\sigma_{L_{\mathbf{x}_{\mathbf{k}} \rightarrow \mathbf{r}_{\mathbf{r}}}}$ be its mean and standard deviation. Furthermore, let $C_{G_{\mathbf{x}_{\mathbf{k}} \rightarrow \mathbf{r}_{\mathbf{r}}}, G_{\mathbf{x}_{\mathbf{j}} \rightarrow \mathbf{r}_{\mathbf{n}}}}:=\mathbb{E}\left\{\left(G_{\mathbf{x}_{\mathbf{k}} \rightarrow \mathbf{r}_{\mathbf{r}}}-\right.\right.$ $\left.\left.\mu_{G_{\mathbf{x}_{\mathbf{k}} \rightarrow \mathbf{r}_{\mathbf{r}}}}\right)\left(G_{\mathbf{x}_{\mathbf{j}} \rightarrow \mathbf{r}_{\mathbf{n}}}-\mu_{G_{\mathbf{x}_{\mathbf{j}} \rightarrow \mathbf{r}_{\mathbf{n}}}}\right)\right\}$ denote the cross-covariance of $G_{\mathbf{x}_{\mathbf{k}} \rightarrow \mathbf{r}_{\mathbf{r}}}$ and $G_{\mathbf{x}_{\mathbf{j}} \rightarrow \mathbf{r}_{\mathbf{n}}}$ for $(k, r) \neq(j, n)$.

Thus, the r.v. $i_{r}$ can be viewed as a sum of (possibly correlated) log-normal r.v.'s. Since an exact expression for the distribution of a sum of log-normally distributed r.v.'s is still too cumbersome for our purpose, further approximation is pursued. The Fenton-Wilkinson method [11] and the SchwartzYeh method [21] were originally developed to approximate the distribution of a sum of independent log-normal r.v.'s by another log-normal r.v. via a cumulant-matching technique [22]. These methods have been extended to the case of correlated log-normal r.v.'s in [23]. The Fenton-Wilkinson method-based technique is suitable for our purpose due to its accuracy over a wide range of parameters, and the simplicity of the resultant. More recent work has been reported on more elaborate approximation [24], but may be too complex for our optimization framework.

Let $I_{r}:=10 \log _{10} i_{r}$, and consider a Gaussian r.v. $\tilde{I}_{r}$ with mean $\mu_{\tilde{I}_{r}}$ and variance $\sigma_{\tilde{I}_{r}}^{2}$. By matching the first two moments of $i_{r}$ and $\tilde{i}_{r}:=e^{\kappa \tilde{I}_{r}}$, one can determine [23]

$$
\mu_{\tilde{I}_{r}}=\kappa^{-1} \ln \left(\frac{\xi_{I_{r}, 1}}{\xi_{I_{r}, 2}^{1 / 2}}\right), \quad \sigma_{\tilde{I}_{r}}^{2}=\kappa^{-2} \ln \left(\frac{\xi_{I_{r}, 2}}{\xi_{I_{r}, 1}}\right)
$$


where

$$
\begin{aligned}
& \xi_{I_{r}, 1}:=\left(\sum_{k=1}^{K} p_{k} a_{r, k}\right)^{2} \\
& \xi_{I_{r}, 2}:=\sum_{k=1}^{K} p_{k}^{2} b_{r, k}+2 \sum_{k=1}^{K-1} \sum_{j=k+1}^{K} p_{k} p_{j} b_{r, k, j}^{\prime} \\
& a_{r, k}:=e^{\kappa \mu_{G_{\mathbf{x}_{\mathbf{k}} \rightarrow \mathbf{r}_{\mathbf{r}}}}+\frac{\kappa^{2}}{2} \sigma_{G_{\mathbf{x}_{\mathbf{k}} \rightarrow \mathbf{r}_{\mathbf{r}}}^{2}}} \\
& b_{r, k}:=e^{2 \kappa \mu_{G_{\mathbf{x}_{\mathbf{k}} \rightarrow \mathbf{r}_{\mathbf{r}}}}+2 \kappa^{2} \sigma_{G_{\mathbf{x}_{\mathbf{k}} \rightarrow \mathbf{r}_{\mathbf{r}}}^{2}}^{2}}
\end{aligned}
$$

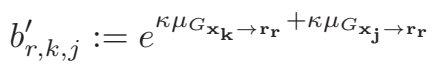

$$
\begin{aligned}
& \cdot e^{\frac{\kappa^{2}}{2}\left(\sigma_{G_{\mathbf{x}_{\mathbf{k}} \rightarrow \mathbf{r}_{\mathbf{r}}}}^{2}+\sigma_{G_{\mathbf{X}_{\mathbf{j}} \rightarrow \mathbf{r}_{\mathbf{r}}}}^{2}+2 C_{G_{\mathbf{X}_{\mathbf{k}} \rightarrow \mathbf{r}_{\mathbf{r}}}, G_{\mathbf{X}_{\mathbf{j}} \rightarrow \mathbf{r}_{\mathbf{r}}}}\right)} .
\end{aligned}
$$

Thus, given the first- and the second-order statistics of the shadow fading in $\mathrm{dB}$ scale, and the $m$ parameter of the Nakagami- $m$-distributed small-scale fading, the distribution of the dB-scale interference $I_{r}$ at PU $r$ can be approximated as Gaussian with mean and variance given in terms of the optimization variables collected in the vector $\mathbf{p}$.

Based on the foregoing discussion, and with $I_{r}^{\max }:=$ $10 \log _{10} i_{r}^{\max }$, the interference constraints in (5c) can be approximated by

$$
\operatorname{Pr}\left\{\tilde{I}_{r}(\mathbf{p})>I_{r}^{\max }\right\} \approx Q\left(\frac{I_{r}^{\max }-\mu_{\tilde{I}_{r}}(\mathbf{p})}{\sigma_{\tilde{I}_{r}}(\mathbf{p})}\right) \leq \epsilon_{r},
$$

$r=1,2, \ldots, R$, where $Q(x):=\int_{x}^{\infty} \frac{1}{\sqrt{2 \pi}} e^{-\frac{t^{2}}{2}} d t$ denotes the Gaussian tail function. Constraints (13) can be equivalently written as

$$
\mu_{\tilde{I}_{r}}(\mathbf{p})+Q^{-1}\left(\epsilon_{r}\right) \sigma_{\tilde{I}_{r}}(\mathbf{p}) \leq I_{r}^{\max }, \quad r=1,2, \ldots, R .
$$

Remark 1. Assuming the knowledge of path loss, shadowing, and small-scale fading distributions is common in the literature [4], [25]-[27]. The channel gain map-based algorithm [3], [5] is an example of how such statistics may be acquired. In some cases, the channel statistics can be obtained from local databases such as the ones used by cellular network operators. However, our proposed approach does not depend on particular estimation methods. Mobility is one of the many causes that make channel estimation challenging. Our optimization framework can accommodate channel uncertainty due to mobility, as long as it is captured in the statistical model of the channel. In the case of low mobility, important parameters such as the $m$ parameter of Nakagami fading may be tracked. Alternatively, a robust approach can be taken [28]: if $m$ is known to be within an interval $\left[m^{\min }, m^{\max }\right]$, a conservative constraint can be imposed by using the worstcase value of $m=m^{\text {min }}$ to ensure robust PU protection. Nevertheless, mobility modeling and assessing its influence on the performance is beyond the scope of this work.

\section{Sequential GP Solution}

\section{A. Problem Re-formulation}

Substituting (11) into (14) still does not lead to a tractable formulation. Instead, by introducing a set of positive auxiliary variables $\mathbf{z}_{r}:=\left[z_{r, 1}, z_{r, 2}\right]^{T}$, with $z_{r, 2}>1, r=1,2, \ldots, R$, the following set of constraints equivalent to (14) is considered:

$$
\begin{aligned}
& \mu_{\tilde{I}_{r}}(\mathbf{p}) \leq \ln \left(z_{r, 1}\right), \quad r=1, \ldots, R \\
& \sigma_{\tilde{I}_{r}}(\mathbf{p}) \leq \ln \left(z_{r, 2}\right), \quad r=1, \ldots, R \\
& \phi_{r}\left(\mathbf{z}_{r}\right):=\ln \left(z_{r, 1}\right)+Q^{-1}\left(\epsilon_{r}\right) \sqrt{\ln \left(z_{r, 2}\right)}-I_{r}^{\max } \leq 0, \\
& r=1, \ldots, R .
\end{aligned}
$$

Using (11), and after some manipulations, it is possible to express (15a) and (15b) as the following ratios of posynomials [29].

$$
\begin{aligned}
& \frac{\xi_{I_{r}, 1}^{2}(\mathbf{p})}{\xi_{I_{r}, 2}(\mathbf{p}) z_{r, 1}^{2 \kappa}} \leq 1, \quad r=1, \ldots, R \\
& \frac{\xi_{I_{r}, 2}(\mathbf{p})}{\xi_{I_{r}, 1}(\mathbf{p}) z_{r, 2}^{\kappa^{2}}} \leq 1, \quad r=1, \ldots, R .
\end{aligned}
$$

The objective functions also need to be re-formulated. Toward this objective, convenient surrogates for the utility functions $\mathcal{U}_{n}$ are derived next for $n \in\{1, \ldots, 4\}$ (see also [17]).

i) Weighted sum-rate utility. Maximizing $\mathcal{U}_{1}$ amounts to minimizing $\prod_{k=1}^{K}\left(1+\gamma_{k}(\mathbf{p})\right)^{-w_{k}}$. When the weights $w_{k}$ are all equal to unity, the method to be described in Sec. IV-B is directly applicable. However, this is not true for general values of $w_{k}$. To handle the latter case, it is convenient to introduce auxiliary variables $\mathbf{t}:=$ $\left[t_{1}, \ldots, t_{K}\right]^{T} \succ \mathbf{0}$. Then, the original weighted sum-rate maximization is equivalent to minimizing

$$
\mathcal{U}_{1}^{\prime}(\mathbf{t})=\prod_{k=1}^{K} t_{k}^{w_{k}}
$$

under the additional constraints $\left[1+\gamma_{k}(\mathbf{p})\right]^{-1} \leq t_{k}, \forall k$, which are equivalent to: [cf. (2)]

$$
\begin{array}{r}
\zeta_{1, k}(\mathbf{p}, \mathbf{t}):=\frac{\sum_{i=1, i \neq k}^{K} p_{k} g_{\mathbf{x}_{\mathbf{i}} \rightarrow \mathbf{u}_{\mathbf{k}}}+\pi_{k}+\sigma_{k}^{2}}{\sum_{i=1}^{K} p_{k} g_{\mathbf{x}_{\mathbf{i}} \rightarrow \mathbf{u}_{\mathbf{k}}}+\pi_{k}+\sigma_{k}^{2}} t_{k}^{-1} \leq 1, \\
k=1, \ldots, K .
\end{array}
$$

ii) Weighted proportional fairness utility. Maximization of $\mathcal{U}_{2}$ is attained equivalently by minimizing $\prod_{k=1}^{K}\left(\log _{2}\left(1+\gamma_{k}(\mathbf{p})\right)^{-w_{k}}\right.$. Then, consider the approximation $\ln (x) \approx q\left(x^{1 / q}-1\right), x>0$ with $q \gg 1$ [29]. Using this approximation and employing auxiliary variables $\mathbf{t}$, yield the following surrogate utility

$$
\mathcal{U}_{2}^{\prime}(\mathbf{t})=\mathcal{U}_{1}^{\prime}(\mathbf{t})
$$

which has to be minimized, under $K$ extra constraints given by [cf. (2)]

$$
\begin{aligned}
& \zeta_{2, k}(\mathbf{p}, \mathbf{t}):= \\
& \left(1+t_{k}^{-1}\right)^{q} \frac{\sum_{i=1, i \neq k}^{K} p_{k} g_{\mathbf{x}_{\mathbf{i}} \rightarrow \mathbf{u}_{\mathbf{k}}}+\pi_{k}+\sigma_{k}^{2}}{\sum_{i=1}^{K} p_{k} g_{\mathbf{x}_{\mathbf{i}} \rightarrow \mathbf{u}_{\mathbf{k}}}+\pi_{k}+\sigma_{k}^{2}} \leq 1, \\
& \quad k=1, \ldots, K .
\end{aligned}
$$

iii) Weighted harmonic-mean-rate utility. Maximizing $\mathcal{U}_{3}$ is equivalent to minimizing function 
$\sum_{k=1}^{K} \frac{1}{w_{k} \log \left(1+\gamma_{k}(\mathbf{p})\right)}$. Approximating the logarithmic function as in the previous case, and again employing auxiliary variables $\mathbf{t}$, maximization of $\mathcal{U}_{3}$ can be achieved by minimizing

$$
\mathcal{U}_{3}^{\prime}(\mathbf{t})=\sum_{k=1}^{K} \frac{t_{k}}{w_{k}}
$$

under constraints (20); that is, $\zeta_{3, k}(\mathbf{p}, \mathbf{t})=\zeta_{2, k}(\mathbf{p}, \mathbf{t})$.

iv) Weighted minimum-rate utility. Note first that maximization of $\mathcal{U}_{4}$ and $\operatorname{minimization} \max _{k}\left[1+\gamma_{k}(\mathbf{p})\right]^{-w_{k}}$ are equivalent. Then, upon introducing an auxiliary variable $\mathbf{t}:=t>0$, maximization of $\mathcal{U}_{4}$ can be achieved by minimizing

$$
\mathcal{U}_{4}^{\prime}(\mathbf{t})=t
$$

under the $K$ extra constraints expressed by means of rational functions of posynomials

$$
\zeta_{4, k}(\mathbf{p}, \mathbf{t}):=\frac{\sum_{i=1, i \neq k}^{K} p_{k} g_{\mathbf{x}_{\mathbf{i}} \rightarrow \mathbf{u}_{\mathbf{k}}}+\pi_{k}+\sigma_{k}^{2}}{\sum_{i=1}^{K} p_{k} g_{\mathbf{x}_{\mathbf{i}} \rightarrow \mathbf{u}_{\mathbf{k}}}+\pi_{k}+\sigma_{k}^{2}} t^{-\frac{1}{w_{k}}} \leq 1,
$$

Based on the preceding discussion, the problem to solve becomes

$$
\min _{\mathbf{p} \succ \mathbf{0},\left\{\mathbf{z}_{r} \succ \mathbf{0}\right\}, \mathbf{t} \succ \mathbf{0}} \mathcal{U}_{n}^{\prime}(\mathbf{t})
$$

$$
\begin{aligned}
& \text { subject to } \zeta_{n, k}(\mathbf{p}, \mathbf{t}) \leq 1, \quad k=1, \ldots, K \\
& p_{k} \leq p_{k}^{\max }, \quad k=1, \ldots, K \\
& \frac{\xi_{I_{r}, 1}^{2}(\mathbf{p})}{\xi_{I_{r}, 2}(\mathbf{p}) z_{r, 1}^{2 \kappa}} \leq 1, \quad r=1, \ldots, R \\
& \frac{\xi_{I_{r}, 2}(\mathbf{p})}{\xi_{I_{r}, 1}(\mathbf{p}) z_{r, 2}^{\kappa^{2}}} \leq 1, \quad r=1, \ldots, R \\
& \phi_{r}\left(\mathbf{z}_{r}\right) \leq 0, \quad r=1, \ldots, R .
\end{aligned}
$$

Problem (P4) involves constraints expressed as ratios of posynomials in (24b), (24d), and (24e), which are non-convex. Another source of non-convexity is (24f). Thus, it is in general difficult to obtain a globally optimal solution to (P4). Recall that this is true even for the case of perfect channel knowledge in (P1) [12]. Therefore, we resort to a successive convex approximation method to obtain (at least locally) optimal solutions [30]. Interestingly, it will be shown that this approach boils down to a sequential GP as in the perfect channel knowledge case [12].

Note that the present work assumes that the set of CRs allowed to transmit at the same time is already determined, and performs power control for the scheduled links. A scheduler design is reported in [31].

Remark 2. In [10], a rate maximization problem under peak interference constraints is considered for a single CR and a single PU. However, the work captures only the uncertainty in small-scale fading, while tacitly assuming that shadow fading is perfectly known. Due to infrequent PU transmissions, or when trying to protect $\mathrm{PU}$ receivers that are silent, the CR might be unable to accurately estimate shadowing, let alone small-scale fading. Thus, [10] and our work are complementary. Incidentally, our formulation admits a closed-form solution in the case of a single CR, given by: $p_{1}=\min \left\{p_{1}^{\max }, e^{\kappa P_{1}}\right\}$, where $P_{1}=\min \left\{I_{r}^{\max }-\mu_{G_{\mathbf{x}_{1} \rightarrow \mathbf{r}_{r}}}-\right.$ $\left.Q^{-1}\left(\epsilon_{r}\right) \sigma_{G_{\mathbf{x}_{1} \rightarrow \mathbf{r}_{r}}}\right\}_{r=1}^{R}$.

\section{B. Successive Convex Approximation}

Here, the general successive convex approximation method is briefly described [30]. Consider an optimization problem

$$
\begin{gathered}
\min _{\mathbf{p} \in \mathcal{P}} f_{0}(\mathbf{p}) \\
\text { subject to } f_{k}(\mathbf{p}) \leq 0, \quad k=1,2, \ldots, K
\end{gathered}
$$

where $f_{0}(\mathbf{p})$ is convex and differentiable, $f_{k}(\mathbf{p}), k=$ $1, \ldots, K$, are differentiable functions, and the feasible region $\mathcal{F}:=\left\{\mathbf{p} \in \mathcal{P} \mid f_{k}(\mathbf{p}) \leq 0, k=1, \ldots, K\right\}$ is compact. Then, starting from a feasible point $\mathbf{p}^{(0)} \in \mathcal{F}$, a series of approximate problems can be solved to locate a KKT point of the original (non-convex) problem. For each $k=1, \ldots, K$, let $\tilde{f}_{k}\left(\mathbf{p} ; \mathbf{p}^{(j)}\right)$ denote the surrogate function for $f_{k}(\mathbf{p})$, which may depend on the solution $\mathbf{p}^{(j)}$ to the problem of the $(j-1)$ st iteration. The approximate problem to solve in iteration $j$ is

$$
\begin{aligned}
& \min _{\mathbf{p} \in \mathcal{P}} f_{0}(\mathbf{p}) \\
\text { subject to } & \tilde{f}_{k}\left(\mathbf{p} ; \mathbf{p}^{(j)}\right) \leq 0, \quad k=1,2, \ldots, K
\end{aligned}
$$

whose feasible region is denoted as $\mathcal{F}^{(j)}$. Provided that $\tilde{f}_{k}\left(\mathbf{p} ; \mathbf{p}^{(j)}\right)$ satisfies the following conditions $\left.\left.\mathbf{c} 1\right)-\mathrm{c} 3\right)$ for each $k=1, \ldots, K$, the series of solutions $\mathbf{p}^{(j)}, j=1,2, \ldots$, to the approximate problems converges to the KKT point of the original problem:
c1) $f_{k}(\mathbf{p}) \leq \tilde{f}_{k}\left(\mathbf{p} ; \mathbf{p}^{(j)}\right), \quad \forall \mathbf{p} \in \mathcal{F}^{(j)}$
c2) $f_{k}\left(\mathbf{p}^{(j)}\right)=\tilde{f}_{k}\left(\mathbf{p}^{(j)} ; \mathbf{p}^{(j)}\right)$
c3) $\nabla f_{k}\left(\mathbf{p}^{(j)}\right)=\nabla \tilde{f}_{k}\left(\mathbf{p}^{(j)} ; \mathbf{p}^{(j)}\right)$.

\section{Sequential GP}

In order to apply the successive convex approximation method to (P4), appropriate surrogate constraints for the nonconvex constraints need to be determined. For the ratio of posynomials in (24b), (24d), and (24e), the single condensation method [32] is adopted for simplicity.

In the single condensation method, each ratio-ofposynomials constraint is approximated by a posynomial constraint. Specifically, a constraint given by

$$
\frac{\sum_{\ell} n_{\ell}(\mathbf{p})}{\sum_{\ell} d_{\ell}(\mathbf{p})} \leq 1
$$

with monomials $\left\{n_{\ell}(\mathbf{p})\right\}$ and $\left\{d_{\ell}(\mathbf{p})\right\}$ is approximated as

$$
\frac{\sum_{\ell} n_{\ell}(\mathbf{p})}{\prod_{\ell}\left(\frac{d_{\ell}(\mathbf{p})}{\alpha_{\ell}}\right)^{\alpha_{\ell}}} \leq 1
$$

where $\alpha_{\ell}:=d_{\ell}\left(\mathbf{p}^{(j)}\right) / \sum_{\ell^{\prime}} d_{\ell^{\prime}}\left(\mathbf{p}^{(j)}\right)$ for a given point $\mathbf{p}^{(j)}$. Then, by viewing the left hand sides (1.h.s.'s) of (29) and (30) as $f_{k}(\mathbf{p})$ and $\tilde{f}_{k}\left(\mathbf{p} ; \mathbf{p}^{(j)}\right)$, respectively, it can be readily shown that they satisfy conditions c1)-c3) in Sec. IV-B [12].

To handle the non-convexity in (24f), it is first noted that $\phi_{r}\left(\mathbf{z}_{r}\right)$ is a concave function for $\mathbf{z}_{r} \succ \mathbf{0}$, which can be easily verified by examining the second-order derivatives of each term [cf. (15c)], and recalling that the sum of concave 
functions is concave. Thus, an upper-bound of $\phi_{r}\left(\mathbf{z}_{r}\right)$ that satisfies c1)-c3) can be obtained as a supporting hyperplane given by

$$
\begin{aligned}
\tilde{\phi}_{r}\left(\mathbf{z}_{r} ; \mathbf{z}_{r}^{(j)}\right) & :=\frac{z_{r, 1}}{z_{r, 1}^{(j)}}+\frac{Q^{-1}\left(\epsilon_{r}\right) z_{r, 2}}{2 z_{r, 2}^{(j)} \sqrt{\ln z_{r, 2}^{(j)}}}-c_{r}\left(\mathbf{z}_{r}^{(j)}\right) \\
c_{r}\left(\mathbf{z}_{r}^{(j)}\right) & :=1+\frac{Q^{-1}\left(\epsilon_{r}\right)}{2 \sqrt{\ln z_{r, 2}^{(j)}}}-\phi_{r}\left(\mathbf{z}_{r}^{(j)}\right) .
\end{aligned}
$$

It is apparent from (32) and (24f) that $c_{r}\left(\mathbf{z}_{r}^{(j)}\right)>0$ provided that $\mathbf{z}_{r}^{(j)}$ is a feasible point, and $\epsilon_{r}<0.5$. Therefore, a surrogate constraint for (24f) is

$$
\frac{1}{c_{r}\left(\mathbf{z}_{r}^{(j)}\right)}\left(\tilde{\phi}_{r}\left(\mathbf{z}_{r} ; \mathbf{z}_{r}^{(j)}\right)+c_{r}\left(\mathbf{z}_{r}^{(j)}\right)\right) \leq 1, r=1, \ldots, R .
$$

The l.h.s. is an affine function with positive coefficients, and thus it is a posynomial.

Overall, the problem to solve in the $j$-th successive iteration is given by (P4) with constraints (24b) and (24d) (24e) replaced by their surrogates per (30), as well as (24f) substituted with (33). It is immediate that this problem is a GP problem, which involves minimizing a posynomial subject to posynomial inequality constraints. Although GP problems are not convex in their original form, their globally optimal solution can be obtained by convex re-formulation through a log change of variables [29]. GP problems can be solved efficiently through optimized interior-point methods.

From a practical perspective, the sequential GP algorithm requires a stopping criterion. Upon defining an error tolerance $v>0$, a simple stopping rule consists in checking whether condition $\mathcal{U}_{n}^{\prime}\left(\mathbf{t}^{(j-1)}\right)-\mathcal{U}_{n}^{\prime}\left(\mathbf{t}^{(j)}\right) \leq v$ is satisfied.

Remark 3. (Average interference constraint) Constraint $(5 \mathrm{c})$ limits the probability that the instantaneous (peak) interference inflicted to a PU receiver exceeds a given threshold $i_{r}^{\max }$. An alternative way of limiting interference is to constrain the average interference power [6], [33], [34]. Consider the approximated constraints $\mathbb{E}\left\{\tilde{i}_{r}\right\} \leq i_{r}^{\max }, r=1, \ldots, R$. Since $\mathbb{E}\left\{\tilde{i}_{r}\right\}=\exp \left(\kappa \mu_{\tilde{I}_{r}}+\frac{\kappa^{2}}{2} \sigma_{\tilde{I}_{r}}^{2}\right)$, the latter constraints are equivalent to

$$
\mu_{\tilde{I}_{r}}(\mathbf{p})+\frac{\kappa}{2} \sigma_{\tilde{I}_{r}}^{2}(\mathbf{p}) \leq I_{r}^{\max }, \quad r=1,2, \ldots, R .
$$

Since (34) can be re-expressed equivalently via the set of constraints (16a), (16b), and

$$
\phi_{r}^{\prime}\left(\mathbf{z}_{r}\right):=\ln \left(z_{r, 1}\right)+\frac{\kappa}{2} \ln \left(z_{r, 2}\right)-I_{r}^{\max } \leq 0, \quad r=1, \ldots, R
$$

with $z_{r, 2}>1$, the successive GP method can be readily applied, using an affine approximation to the concave function $\phi_{r}^{\prime}\left(\mathbf{z}_{r}\right)$ around $\mathbf{z}_{r}^{(j)}$; that is, $\tilde{\phi}_{r}(\cdot)$ and $c_{r}(\cdot)$ in (31)-(33) are replaced by $\tilde{\phi}_{r}^{\prime}\left(\mathbf{z}_{r} ; \mathbf{z}_{r}^{(j)}\right):=\frac{z_{r, 1}}{z_{r, 1}^{(j)}}+\frac{\kappa}{2 z_{r, 2}^{(j)}} z_{r, 2}-c_{r}^{\prime}\left(\mathbf{z}_{r}^{(j)}\right)$ and $c_{r}^{\prime}\left(\mathbf{z}_{r}^{(j)}\right):=1+\frac{\kappa}{2}-\phi_{r}^{\prime}\left(\mathbf{z}_{r}^{(j)}\right)$, respectively.

\section{UNCERTAIN CR-TO-CR CHANNELS}

Problem (P3) incorporates CR-to-CR channel uncertainty by introducing outage probability constraints on a per-CR link SINRs, in addition to the PU protection outage constraints that mitigate CR-to-PU channel uncertainty. In order to obtain a tractable solution method, the outage constraint (6d) needs to be re-written in a closed form in terms of the variables $\mathbf{p}$ and $\bar{\gamma}$.

\section{A. Approximation of SINR Distributions}

First, express $\gamma_{k}^{-1}, \quad k=1, \ldots, K$, as $\gamma_{k}^{-1}=$ $\sum_{j=1, j \neq k}^{K} p_{k}^{-1} p_{j} g_{\mathbf{x}_{\mathbf{k}} \rightarrow \mathbf{u}_{\mathbf{k}}}^{-1} g_{\mathbf{x}_{\mathbf{j}} \rightarrow \mathbf{u}_{\mathbf{k}}}+p_{k}^{-1} g_{\mathbf{x}_{\mathbf{k}} \rightarrow \mathbf{u}_{\mathbf{k}}}^{-1}\left(\pi_{k}+\sigma_{k}^{2}\right)$ and notice that $10 \log _{10}\left(p_{k}^{-1} p_{j} g_{\mathbf{x}_{\mathbf{k}} \rightarrow \mathbf{u}_{\mathbf{k}}}^{-1} g_{\mathbf{x}_{\mathbf{j}} \rightarrow \mathbf{u}_{\mathbf{k}}}\right)$ is Gaussian with mean and variance given by $-P_{k}+P_{j}-\mu_{G_{\mathbf{x}_{\mathbf{k}} \rightarrow \mathbf{u}_{\mathbf{k}}}}+\mu_{G_{\mathbf{x}_{\mathbf{j}} \rightarrow \mathbf{u}_{\mathbf{k}}}}$, and $\sigma_{G_{\mathbf{x}_{\mathbf{k}} \rightarrow \mathrm{u}_{\mathrm{k}}}}^{2}+\sigma_{G_{\mathbf{x}_{\mathbf{j}} \rightarrow \mathrm{u}_{\mathbf{k}}}}^{2}-2 C_{G_{\mathbf{x}_{\mathbf{k}} \rightarrow \mathbf{u}_{\mathbf{k}}}, G_{\mathbf{x}_{\mathbf{j}} \rightarrow \mathbf{u}_{\mathbf{k}}}}$, respectively. The $\mathrm{dB}$ version of the second term, $10 \log _{10} p_{k}^{-1} g_{\mathbf{x}_{\mathbf{k}} \rightarrow \mathbf{u}_{\mathbf{k}}}^{-1}\left(\pi_{k}+\right.$ $\left.\sigma_{k}^{2}\right)$, is also Gaussian, with mean $-P_{k}-\mu_{G_{\mathbf{x}_{\mathbf{k}} \rightarrow \mathbf{u}_{\mathbf{k}}}}+$ $10 \log _{10}\left(\pi_{k}+\sigma_{k}^{2}\right)$ and variance $\sigma_{G_{\mathbf{x}_{\mathbf{k}} \rightarrow \mathbf{u}_{\mathbf{k}}}}^{2}$. Thus, $\gamma_{k}^{-1}$ involves summation of correlated log-normal r.v.'s. Again, the FentonWilkinson method can be employed to find a log-normal approximation of $\gamma_{k}^{-1}$ and, subsequently, of $\gamma_{k}$.

Let $\Gamma_{k}:=10 \log _{10} \gamma_{k}$, and consider a Gaussian r.v. $\tilde{\Gamma}_{k}:=$ $10 \log _{10} \tilde{\gamma}_{k}$ with mean $\mu_{\tilde{\Gamma}_{k}}$ and variance $\sigma_{\tilde{\Gamma}_{k}}^{2}$. By matching the first two moments of $\gamma_{k}^{-1}$ and $\tilde{\gamma}_{k}^{-1}=e^{-\kappa \tilde{\Gamma}_{k}}$, one can establish

$$
\mu_{\tilde{\Gamma}_{k}}=\kappa^{-1} \ln \left(\frac{\xi_{\tilde{\Gamma}_{k}, 2}^{1 / 2}}{\xi_{\tilde{\Gamma}_{k}, 1}}\right), \quad \sigma_{\tilde{\Gamma}_{k}}^{2}=\kappa^{-2} \ln \left(\frac{\xi_{\tilde{\Gamma}_{k}, 2}}{\xi_{\tilde{\Gamma}_{k}, 1}}\right)
$$

where

$$
\begin{aligned}
\xi_{\tilde{\Gamma}_{k}, 1}:= & \left(\sum_{j=1, j \neq k}^{K} p_{k}^{-1} p_{j} \alpha_{k, j}+p_{k}^{-1} \alpha_{k, K+1}\right)^{2} \\
\xi_{\tilde{\Gamma}_{k}, 2}:= & \sum_{\substack{j=1 \\
j \neq k}}^{K} p_{k}^{-2} p_{j}^{2} \beta_{k, j}+p_{k}^{-2} \beta_{k, K+1} \\
& +2 \sum_{\substack{j=1 \\
j \neq k}}^{K-1} \sum_{\substack{i=j+1 \\
i \neq k}}^{K} p_{k}^{-2} p_{j} p_{i} \beta_{k, j, i}^{\prime}+2 \sum_{\substack{j=1 \\
j \neq k}}^{K-1} p_{k}^{-2} p_{j} \beta_{k, j, K+1}^{\prime \prime}
\end{aligned}
$$

and

$$
\begin{aligned}
& \alpha_{k, j}:=e^{\kappa\left(\mu_{\left.G_{\mathbf{x}_{\mathbf{j}} \rightarrow \mathbf{u}_{\mathbf{k}}}-\mu_{G_{\mathbf{x}_{\mathbf{k}}} \rightarrow \mathbf{u}_{\mathbf{k}}}\right)}\right.} \\
& \cdot e^{\frac{\kappa^{2}}{2}\left(\sigma_{G_{\mathbf{x}_{\mathbf{j}} \rightarrow \mathbf{u}_{\mathbf{k}}}^{2}}^{2}+\sigma_{G_{\mathbf{x}_{\mathbf{k}}} \rightarrow \mathbf{u}_{\mathbf{k}}}^{2}-2 C_{G_{\mathbf{x}_{\mathbf{j}}} \rightarrow \mathbf{u}_{\mathbf{k}}}, G_{\mathbf{x}_{\mathbf{k}} \rightarrow \mathbf{u}_{\mathbf{k}}}\right)} \\
& \alpha_{k, K+1}:=\left(\pi_{k}+\sigma_{k}^{2}\right) e^{-\kappa \mu_{G_{\mathbf{x}_{\mathbf{k}}} \rightarrow \mathbf{u}_{\mathbf{k}}}+\frac{\kappa^{2}}{2} \sigma_{G_{\mathbf{x}_{\mathbf{k}}} \rightarrow \mathbf{u}_{\mathbf{k}}}^{2}}
\end{aligned}
$$

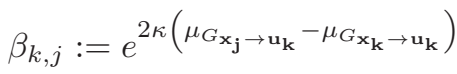

$$
\begin{aligned}
& \cdot e^{2 \kappa^{2}\left(\sigma_{G_{\mathbf{x}_{\mathbf{j}}} \rightarrow \mathbf{u}_{\mathbf{k}}}^{2}+\sigma_{G_{\mathbf{x}_{\mathbf{k}}} \rightarrow \mathbf{u}_{\mathbf{k}}}^{2}-2 C_{G_{\mathbf{x}_{\mathbf{j}} \rightarrow \mathbf{u}_{\mathbf{k}}}, G_{\mathbf{x}_{\mathbf{k}} \rightarrow \mathbf{u}_{\mathbf{k}}}}\right)} \\
& \beta_{k, K+1}:=\left(\pi_{k}+\sigma_{k}^{2}\right)^{2} e^{-2 \kappa \mu_{G_{\mathbf{x}_{\mathbf{k}} \rightarrow \mathbf{u}_{\mathbf{k}}}}+2 \kappa^{2} \sigma_{G_{\mathbf{x}_{\mathbf{k}}} \rightarrow \mathbf{u}_{\mathbf{k}}}^{2}}
\end{aligned}
$$




$$
\begin{aligned}
& \beta_{k, j, i}^{\prime}:=e^{\kappa\left(\mu_{G_{\mathbf{x}_{\mathbf{j}} \rightarrow \mathbf{u}_{\mathbf{k}}}}+\mu_{G_{\mathbf{x}_{\mathbf{i}} \rightarrow \mathbf{u}_{\mathbf{k}}}}-2 \mu_{G_{\mathbf{x}_{\mathbf{k}} \rightarrow \mathbf{u}_{\mathbf{k}}}}\right)} \\
& \cdot e^{\frac{\kappa^{2}}{2}\left(\sigma_{G_{\mathbf{x}_{\mathbf{j}}} \rightarrow \mathbf{u}_{\mathbf{k}}}^{2}+\sigma_{G_{\mathbf{x}_{\mathbf{i}}} \rightarrow \mathbf{u}_{\mathbf{k}}}^{2}+4 \sigma_{G_{\mathbf{x}_{\mathbf{k}}} \rightarrow \mathbf{u}_{\mathbf{k}}}^{2}-4 C_{G_{\mathbf{x}_{\mathbf{j}} \rightarrow \mathbf{u}_{\mathbf{k}}}, G_{\mathbf{x}_{\mathbf{k}} \rightarrow \mathbf{u}_{\mathbf{k}}}}\right.}
\end{aligned}
$$

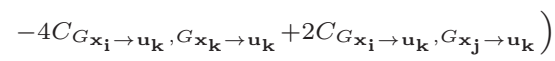

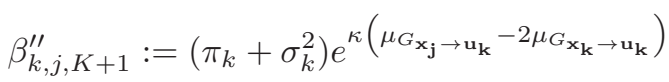

$$
\begin{aligned}
& \cdot e^{\frac{\kappa^{2}}{2}\left(\sigma_{G_{\mathbf{x}_{\mathbf{j}} \rightarrow \mathbf{u}_{\mathbf{k}}}^{2}}^{2}+4 \sigma_{G_{\mathbf{x}_{\mathbf{k}} \rightarrow \mathbf{u}_{\mathbf{k}}}}^{2}-4 C_{G_{\mathbf{x}_{\mathbf{j}} \rightarrow \mathbf{u}_{\mathbf{k}}}, G_{\mathbf{x}_{\mathbf{k}} \rightarrow \mathbf{u}_{\mathbf{k}}}}\right)} .
\end{aligned}
$$

(39f)

Thus, SINRs $\left\{\Gamma_{k}\right\}$ in $\mathrm{dB}$ can be approximated as Gaussian, with mean and variance given as functions of $\mathbf{p}$, provided that $\left\{\mu_{G_{\mathbf{x}_{\mathbf{i}} \rightarrow \mathbf{u}_{\mathbf{k}}}}\right\},\left\{\sigma_{G_{\mathbf{x}_{\mathbf{i}} \rightarrow \mathbf{u}_{\mathbf{k}}}}^{2}\right\}$ and $\left\{C_{G_{\mathbf{x}_{\mathbf{j}} \rightarrow \mathbf{u}_{\mathbf{k}}}}, G_{\mathbf{x}_{\mathbf{i}} \rightarrow \mathbf{u}_{\mathbf{k}}}\right\}$ are known.

Using the log-normal-approximated $\left\{\tilde{\gamma}_{k}\right\}$ in place of $\left\{\gamma_{k}\right\},(6 \mathrm{~d})$ can be approximated as

$$
\operatorname{Pr}\left\{\tilde{\gamma}_{k}(\mathbf{p})<\bar{\gamma}_{k}\right\}=Q\left(\frac{\mu_{\tilde{\Gamma}_{k}}(\mathbf{p})-\kappa^{-1} \ln \left(\bar{\gamma}_{k}\right)}{\sigma_{\tilde{\Gamma}_{k}}(\mathbf{p})}\right) \leq \nu_{k},
$$

$k=1, \ldots, K$, which can be equivalently re-written as

$$
Q^{-1}\left(\nu_{k}\right) \sigma_{\tilde{\Gamma}_{k}}(\mathbf{p})-\mu_{\tilde{\Gamma}_{k}}(\mathbf{p})+\kappa^{-1} \ln \left(\bar{\gamma}_{k}\right) \leq 0, k=1, \ldots, K .
$$

\section{B. Solution Approach}

Following an approach similar to the one in Section IV-A, consider the following set of constraints equivalent to (41) [cf. (15a)-(15c)]:

$$
\begin{aligned}
&-\mu_{\tilde{\Gamma}_{k}}(\mathbf{p}) \leq \ln \left(y_{k, 1}\right), \quad r=1, \ldots, R \\
& \sigma_{\tilde{\Gamma}_{k}}^{2}(\mathbf{p}) \leq \ln \left(y_{k, 2}\right), \quad r=1, \ldots, R \\
& \psi_{k}\left(\mathbf{y}_{k}, \bar{\gamma}_{k}\right):=\ln \left(y_{k, 1}\right)+Q^{-1}\left(\nu_{k}\right) \sqrt{\ln \left(y_{k, 2}\right)}+\frac{1}{\kappa} \ln \left(\bar{\gamma}_{k}\right) \leq 0 \\
& k=1, \ldots, K .
\end{aligned}
$$

where auxiliary variables $\mathbf{y}_{k}:=\left[y_{k, 1}, y_{k, 2}\right]^{T}, k=1, \ldots, K$ have been introduced. Then, using (36), constraints (42)-(43) can be re-written as

$$
\begin{aligned}
& \frac{\xi_{\tilde{\Gamma}_{k}, 1}^{2}(\mathbf{p})}{\xi_{\tilde{\Gamma}_{k}, 2}(\mathbf{p}) y_{k, 1}^{2 \kappa}} \leq 1, \quad k=1, \ldots, K \\
& \frac{\xi_{\tilde{\Gamma}_{k}, 2}(\mathbf{p})}{\xi_{\tilde{\Gamma}_{k}, 1}(\mathbf{p}) y_{k, 2}^{\kappa^{2}}} \\
& \leq 1, \quad k=1, \ldots, K
\end{aligned}
$$

which are ratios of posynomials.

As in the previous section, to obtain the sequential GP-based solution, the objective function of (P3) must be reformulated too. To this end, define the auxiliary vector $\mathbf{t}:=\left[t_{1}, \ldots, t_{K}\right]^{T}$ when one of the utilities $\mathcal{U}_{n}^{\prime}, n \in\{1,2,3\}$, is used and as $\mathbf{t}:=[t]$ when $n=4$.

i) Weighted sum-rate utility. Following the procedure in Section IV-A, maximization of the sum-outage-rate $\sum_{k} w_{k} \log _{2}\left(1+\bar{\gamma}_{k}\right)$ can be equivalently attained upon minimizing cost (17) with $K$ extra constraints

$$
\chi_{1, k}\left(\bar{\gamma}_{k}, \mathbf{t}\right):=\frac{1}{\left(1+\bar{\gamma}_{k}\right) t_{k}} \leq 1, \quad k=1, \ldots, K .
$$

ii) Weighted proportional fairness utility. Utility $\sum_{k=1}^{K} w_{k} \log _{2}\left(\log _{2}\left(1+\bar{\gamma}_{k}\right)\right)$ is maximized upon minimizing (19), subject to

$$
\chi_{2, k}\left(\bar{\gamma}_{k}, \mathbf{t}\right)=\frac{\left(1+t_{k}^{-1}\right)^{q}}{1+\bar{\gamma}_{k}} \leq 1 .
$$

iii) Weighted harmonic-mean-rate utility. Based on the foregoing re-formulations, maximization of the harmonic-outage-rate utility can be attained by minimizing (21) under constraints (48); that is, $\chi_{3, k}\left(\bar{\gamma}_{k}, \mathbf{t}\right)=$ $\chi_{2, k}\left(\bar{\gamma}_{k}, \mathbf{t}\right)$.

iv) Weighted minimum-rate utility. Maximization of $\min _{k} \log _{2}\left(1+\bar{\gamma}_{k}\right)$ can be attained by minimizing (22) under the following $K$ extra constraints

$$
\chi_{4, k}\left(\bar{\gamma}_{k}, \mathbf{t}\right)=\left(1+\bar{\gamma}_{k}\right)^{-1} t^{-\frac{1}{w_{k}}} \leq 1, \quad k=1, \ldots, K .
$$

Then, based on the preceding discussion, a substitute for problem (P3) can be written as:

(P5)

$$
\min _{\substack{\mathbf{p} \succ \mathbf{0} \\\left\{\mathbf{z}_{r} \succ \mathbf{0}\right\},\left\{\mathbf{y}_{k} \succ \mathbf{0}\right\} \\ \overline{\boldsymbol{\gamma}} \succ \mathbf{0}, \mathbf{t} \succ \mathbf{0}}} \mathcal{U}_{n}^{\prime}(\mathbf{t})
$$

$$
\text { subject to }
$$

$$
\chi_{n, k}\left(\bar{\gamma}_{k}, \mathbf{t}\right) \leq 1
$$$$
p_{k} \leq p_{k}^{\max }, k=1, \ldots, K
$$$$
\frac{\xi_{\tilde{\Gamma}_{k}, 1}^{2}(\mathbf{p})}{\xi_{\tilde{\Gamma}_{k}, 2}(\mathbf{p}) y_{k, 1}^{2 \kappa}} \leq 1, \quad k=1, \ldots, K
$$

$$
\begin{gathered}
\frac{\xi_{\tilde{\Gamma}_{k}, 2}(\mathbf{p})}{\xi_{\tilde{\Gamma}_{k}, 1}(\mathbf{p}) y_{k, 2}^{\kappa^{2}}} \leq 1, \quad k=1, \ldots, K \\
\psi_{k}\left(\mathbf{y}_{k}, \bar{\gamma}_{k}\right) \leq 0, \quad k=1, \ldots, K \\
\text { and }(24 \mathrm{~d}),(24 \mathrm{e}),(24 \mathrm{f})
\end{gathered}
$$

where $n \in\{1,2,3,4\}$. The sequential GP technique can again be applied by approximating the ratio-of-posynomial constraints (50b), (50d), and (50e) using the single condensation method, and the concave function $\psi_{k}(\cdot)$ in (44) by using its affine upper-bound [cf. (31)]

$$
\begin{aligned}
& \tilde{\psi}_{k}\left(\mathbf{y}_{k}, \bar{\gamma}_{k} ; \mathbf{y}_{k}^{(j)}, \bar{\gamma}_{k}^{(j)}\right):= \\
& \quad \frac{y_{k, 1}}{y_{k, 1}^{(j)}}+\frac{y_{k, 2} Q^{-1}\left(\nu_{k}\right)}{2 y_{k, 2}^{(j)} \sqrt{\ln \left(y_{k, 2}^{(j)}\right)}}+\frac{\bar{\gamma}_{k}}{\kappa \bar{\gamma}_{k}^{(j)}}-c_{y, k}\left(\mathbf{y}_{k}^{(j)}, \bar{\gamma}_{k}^{(j)}\right)
\end{aligned}
$$

with

$$
c_{y, k}\left(\mathbf{y}_{k}^{(j)}, \bar{\gamma}_{k}^{(j)}\right)=\left(1+\kappa^{-1}\right)+\frac{Q^{-1}\left(\nu_{k}\right)}{2 \sqrt{\ln \left(y_{k, 2}^{(j)}\right)}}-\phi_{y, k}\left(\mathbf{y}_{k}^{(j)}, \bar{\gamma}_{k}^{(j)}\right) \text {. }
$$

Note from (50f) and (51) that for feasible points $\mathbf{y}_{k}^{(j)}, \bar{\gamma}_{k}^{(j)}$ and for $\nu_{k}<0.5$, it holds that $c_{y, k}\left(\mathbf{y}_{k}^{(j)}, \bar{\gamma}_{k}^{(j)}\right)>0$. Thus, (50f) can be substituted by

$$
\begin{gathered}
\frac{1}{c_{y, k}\left(\mathbf{y}_{k}^{(j)}, \bar{\gamma}_{k}^{(j)}\right)}\left(\tilde{\psi}_{k}\left(\mathbf{y}_{k}, \bar{\gamma}_{k} ; \mathbf{y}_{k}^{(j)}, \bar{\gamma}_{k}^{(j)}\right)+c_{y, k}\left(\mathbf{y}_{k}^{(j)}, \bar{\gamma}_{k}^{(j)}\right)\right) \leq 1 \\
k=1, \ldots, K
\end{gathered}
$$

Overall, the $j$-th iteration of sequential convex optimization entails a GP, where constraints (24d)-(24e), (50b), and (50d)(50e) in (P5) are replaced by their surrogates per (30); constraint (24f) replaced by (33); and constraint (53) is utilized in place of (50f). 


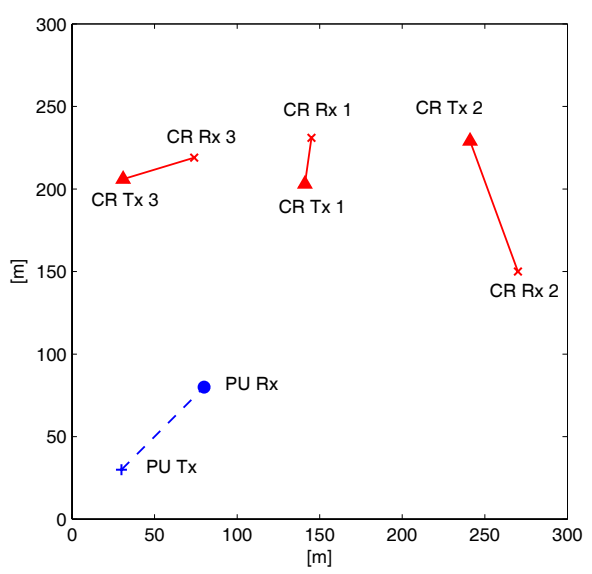

Fig. 1. Test scenario.

\section{Numerical Tests}

Consider the scenario depicted in Fig. 1, involving $K=$ $3 \mathrm{CR}$ links (shown in solid lines), and a $(R=1) \mathrm{PU}$ link (dashed line) being also active with transmit-power $0.1 \mathrm{~W}$. The path loss parameters are set to $g_{k}=1$ for all $k$, and $\eta=$ 3.5 , and $m=10$ is used for Nakagami- $m$ fading. Spatially correlated log-normal shadowing was generated with mean 0 , standard deviation $10 \mathrm{~dB}$, and coherence distance $30 \mathrm{~m}$, based on the model described in [15]. Although the optimized CR transmit-powers turn out to be much lower (in the $\mathrm{mW}$ range), the maximum transmit-power for the $\mathrm{CR}$ system is $p_{k}^{\max }=$ $5 \mathrm{~W}$, while the noise power $\sigma_{k}^{2}=10^{-8}$. The interference threshold was set to $I_{r}^{\max }=-80 \mathrm{dBW}$, and the probability of exceeding the threshold to $\epsilon_{r}=0.01$. Albeit simple, this is a challenging setup for the CRs to obtain sizable rates as the distances between Tx-Crs and the Rx-PU Rx are only 2-3 times the intended transmission distances of the CR links. In order to terminate the sequential GP iterations, a threshold of $v=10^{-4}$ was used.

Consider maximizing the sum-rate of the CR network, with $w_{k}=1$ for all $k$. Suppose first that the CR-to-CR channel gains are perfectly known. To verify the efficacy of the interference constraints, the complementary cumulative distribution function (ccdf) of the received interference power at the PU receiver is plotted in Fig. 2. The curve with square markers corresponds to the case where the PU-to-CR channel statistics were obtained from the a priori knowledge of the node locations, and the shadowing and small-scale fading distributions; c.f. (1). The curve with ' $x$ ' markers represents the case where the shadowing component was estimated with an error standard deviation of $4 \mathrm{~dB}$ (e.g., via channel gain cartography [3]). In these two cases, (P4) was solved to obtain the CR transmission powers. The curves with circles and ' + ' markers depict, respectively, the cases of perfect channel knowledge, and of using a path loss-only model with shadowing and small-scale fading completely neglected (i.e., set to $0 \mathrm{~dB})$. In the last two cases, $(\mathrm{P} 1)$ was solved to obtain optimal powers, and 5,000 independent channel realizations were used to generate the plot.

It is clearly seen in Fig. 2 that solving (P4) enforces the

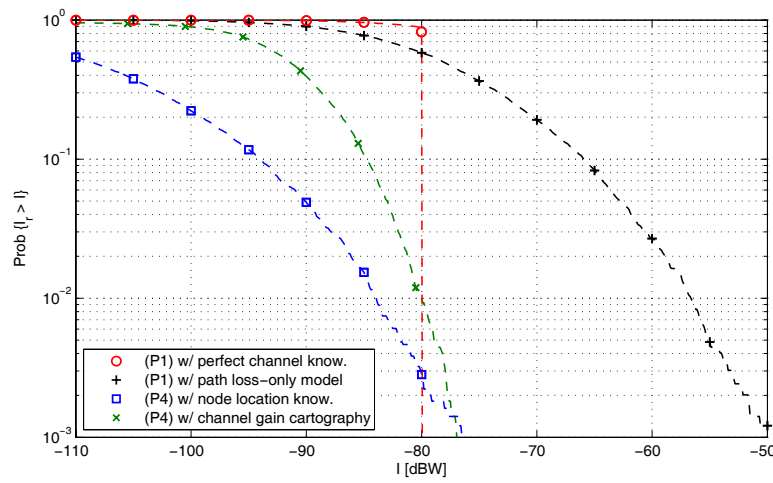

Fig. 2. The ccdf of the interference power.

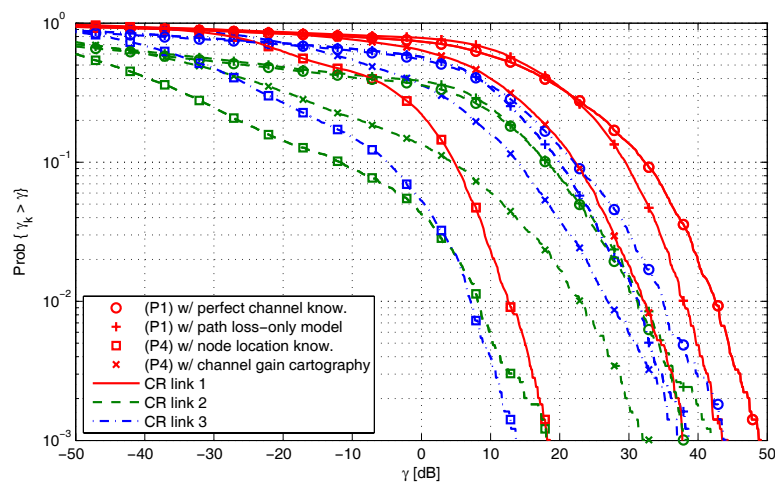

Fig. 3. The ccdf of CR SINRs.

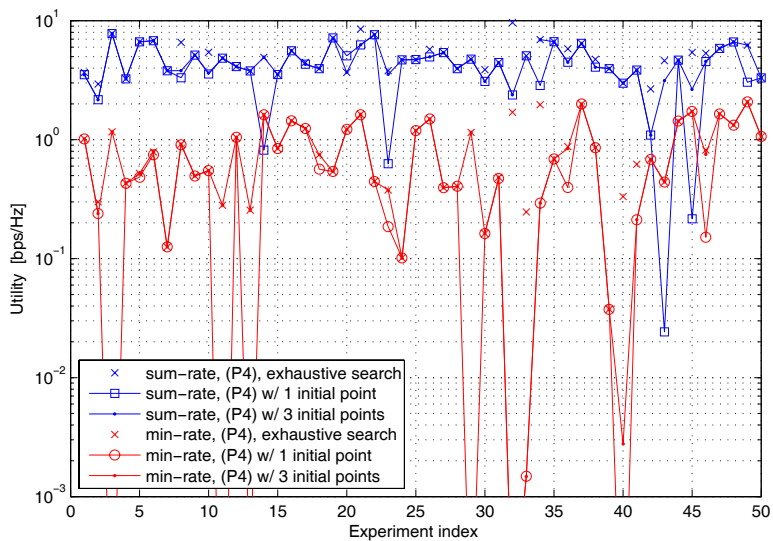

Fig. 4. Achieved network utilities.

probabilistic PU interference constraint. This also verifies the accuracy of the associated approximation techniques. It is interesting to note that without channel gain cartography, the interference constraint is seen to be over-satisfied, while with channel gain cartography, the constraint is tightly met. On the other hand, neglecting the uncertainty in the channel gain results in grossly violating the interference constraint.

Fig. 3 shows the cedf of the SINRs $\left\{\gamma_{k}\right\}$ of the CR links. The solid, dashed, and dash-dotted curves represent different $\mathrm{CR}$ links, and the markers are used in the same manner as 


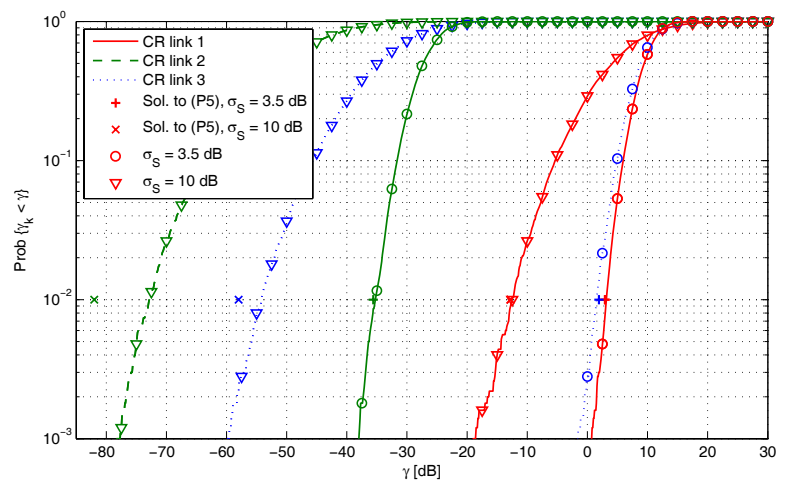

Fig. 5. The c.d.f. of CR SINRs with uncertain CR-to-CR and CR-to-PU channels.

in Fig. 2. It can be seen that when the CR-to-PU channel gain is estimated, the performance is significantly improved compared to the case of using only the location information. The path loss-only model case exhibits better SINRs than the proposed scheme, but only at severe interference to the PU.

To see how close the obtained KKT solutions are to the globally optimal solutions, an exhaustive grid-based search was performed. Fig. 4 depicts the CR sum-rates and the maxmin rates (both with $w_{k}=1$ for all $k$ ) obtained by solving (P4) for 50 different shadowing and small-scale fading realizations. The crosses correspond to the exhaustive search, the solid curves to using sequential GP with a single random initial point, and the dash-dot curves to running sequential GP with three random initial points and retaining the best result. It is seen that the sequential GP-based objectives often coincide with the globally optimal objectives, with using just three initial points significantly enhancing the chance to attain the global optima.

Next, the case of uncertain CR-to-CR and CR-to-PU channels was tested for the scenario in Fig. 1. Different levels of uncertainty were considered by setting the standard deviation of the shadowing to $3.5 \mathrm{~dB}$ and $10 \mathrm{~dB}$. The probability of outage for each CR link was set to $\nu_{k}=0.01$ for all $k$. Problem (P5) was solved using the equally weighted sumrate utility to obtain $\left\{\bar{\gamma}_{k}\right\}$, which is plotted in the figure with ' + ' and ' $\times$ ' markers for $\sigma_{S_{\mathrm{x}_{\mathrm{k}} \rightarrow \mathrm{u}_{\mathrm{k}}}}=3.5 \mathrm{~dB}$ and $\sigma_{S_{\mathrm{x}_{\mathrm{k}} \rightarrow \mathrm{u}_{\mathrm{k}}}}=10 \mathrm{~dB}$, respectively. To verify that the achieved CR link SINRs are indeed within the outage specification, the c.d.f. of the achieved SINRs over different channel realizations are also plotted in Fig. 5. It is seen that the outage constraints are observed for all $\mathrm{CR}$ receivers. One can notice that the lower uncertainty leads to tightly met constraints. Extensive simulation tests confirmed that the outage constraints were always satisfied, verifying the usefulness of the approximation proposed for the SINR statistics. It is also noted that the lower channel gain uncertainty allows the CRs to achieve higher SINRs. The efficacy of the interference constraints is verified also for the case of uncertain CR-to-CR channels, and the ccdf of the received interference power at the PU receiver is plotted in Fig. 6. It is apparent that solving (P5) enforces the probabilistic PU interference constraint.

An exhaustive grid-based search was performed to obtain

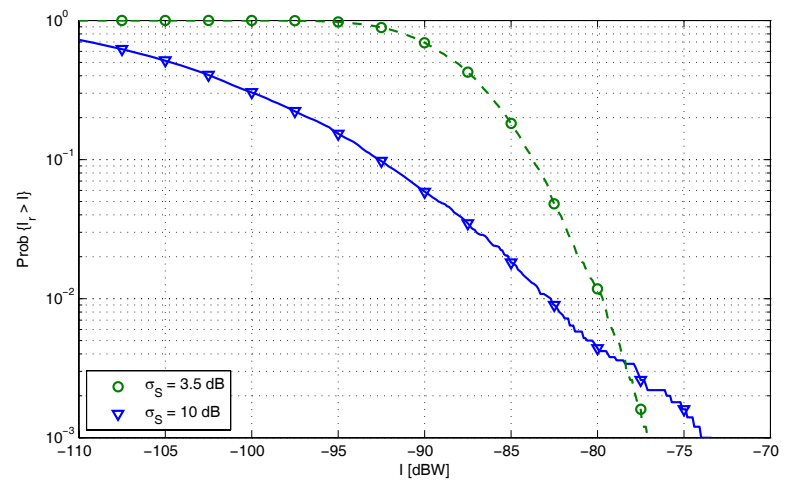

Fig. 6. The ccdf of the interference power with uncertain CR-to-CR and CR-to-PU channels.

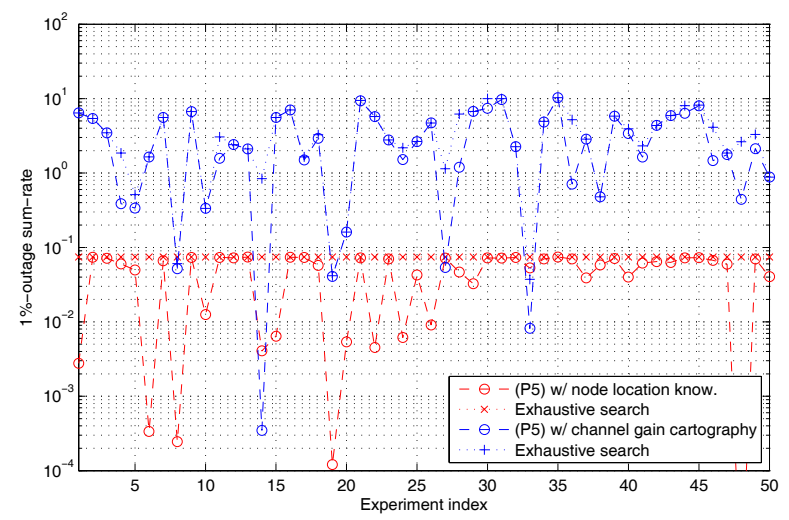

Fig. 7. Achieved sum-outage-rates.

TABLE I

AVERAGE NUMBER OF ITERATIONS REQUiRED TO SOLVE (P1), (P4) AND (P5) VIA SEQUENTIAL GP

\begin{tabular}{l|c|c|c} 
& $(\mathrm{P} 1)$ & $(\mathrm{P} 4)$ & $(\mathrm{P} 5)$ \\
\hline Perfect CR-to-PU and CR-to-CR channel gains & 8 & - & - \\
Path loss-only CR-to-PU and CR-to-CR & 8 & - & - \\
Uncertain channel gains, $\sigma_{S_{\mathbf{x}_{\mathbf{k}}} \rightarrow \mathbf{u}_{\mathbf{k}}}=10 \mathrm{~dB}$ & - & 9 & 26 \\
Uncertain channel gains, $\sigma_{S_{\mathbf{x}_{\mathbf{k}}} \rightarrow \mathbf{u}_{\mathbf{k}}}=3.5 \mathrm{~dB}$ & - & 13 & 36 \\
\hline
\end{tabular}

globally optimal solutions to (P5) with sum-rate utility so as to assess the goodness of the proposed successive GP approach. Two cases are presented: the case of using only the node location information, and the case using channel gain cartography with $4 \mathrm{~dB}$ error standard deviation. In the former case, 50 different (random) initial points were used, while in the latter, the effect of both random initial points and random channel realizations were tested. The $1 \%$-outage constrained sum-rates achieved in the experiments are depicted in Fig. 7. It can be seen that the globally optimal objectives are often achieved by the sequential GP solutions. It is also noticed that significantly higher sum-rates are achieved with channel gain cartography.

To assess the complexity of our algorithm, Table I tabulates the average number of sequential GP iterations necessary until the stopping condition was met. It is seen that the computational burden required to solve (P4) is comparable to 
that of solving (P1), while (P5) requires a considerably higher number of iterations.

\section{CONCLUSIONS}

CR power allocation algorithms were developed under CR-to-PU channel gain uncertainty arising from shadowing and small-scale fading effects. Probabilistic interference constraints were imposed to protect the PU transmissions notwithstanding the channel uncertainty. A Fenton-Wilkinson-type approximation was employed to model the received interference power at the PUs as log-normal, which led to a tractable NUM problem formulation for the CR network. Due to nonconvexity of the problem, a successive convex approximation technique was adopted to obtain a KKT optimal solution. This approach boiled down to a sequential GP algorithm, which can be solved efficiently using, e.g., the interior-point methods. The uncertain CR-to-CR channel case was also considered by introducing an SINR outage-based utility maximization problem. The Fenton-Wilkinson method was again employed to approximate the distribution of the SINRs, and a sequential GP-based solution was proposed. Numerical tests verified the validity and the performance advantages of the proposed schemes.

\section{REFERENCES}

[1] Q. Zhao and B. M. Sadler, "A survey of dynamic spectrum access," IEEE Signal Process. Mag., vol. 24, no. 3, pp. 79-89, May 2007.

[2] J. M. Peha, "Approaches to spectrum sharing," IEEE Commun. Mag., vol. 43, no. 2, pp. 10-12, Feb. 2005.

[3] S.-J. Kim, E. Dall'Anese, and G. Giannakis, "Cooperative spectrum sensing for cognitive radios using Kriged Kalman filtering," IEEE $J$. Sel. Topics Signal Process., vol. 5, no. 1, pp. 24-36, Feb. 2011.

[4] J.-A. Bazerque and G. B. Giannakis, "Distributed spectrum sensing for cognitive radio networks by exploiting sparsity," IEEE Trans. Signal Process., vol. 58, no. 3, pp. 1847-1862, Mar. 2010.

[5] E. Dall'Anese, S.-J. Kim, and G. Giannakis, "Channel gain map tracking via distributed Kriging," IEEE Trans. Veh. Technol., vol. 60, no. 3, pp. 1205-1211, Mar. 2011.

[6] A. Ghasemi and E. S. Sousa, "Fundamental limits of spectrum-sharing in fading environments," IEEE Trans. Wireless Commun., vol. 6, no. 2, pp. 649-658, Feb. 2007.

[7] I. Mitliagkas, N. Sidiropoulos, and A. Swami, "Convex approximationbased joint power and admission control for cognitive underlay networks," in Proc. IEEE IWCMC Conf., 2008, pp. 28-32.

[8] R. Zhang and Y. C. Liang, "Exploiting multi-antennas for opportunistic spectrum sharing in cognitive radio networks," IEEE J. Sel. Topics Signal Process., vol. 2, no. 1, pp. 88-102, Feb. 2008.

[9] A. G. Marques, X. Wang, and G. B. Giannakis, "Dynamic resource management for cognitive radios using limited-rate feedback," IEEE Trans. Signal Process., vol. 57, no. 9, pp. 3651-3666, Sep. 2009.

[10] H. A. Suraweera, P. J. Smith, and M. Shafi, "Capacity limits and performance analysis of cognitive radio with imperfect channel knowledge," IEEE Trans. Veh. Technol., vol. 59, no. 4, pp. 1811-1822, May 2010.

[11] L. F. Fenton, "The sum of lognormal probability distributions in scatter transmission systems," IRE Trans. Commun. Syst., vol. 8, no. 1, pp. 57-67, Mar. 1960.

[12] M. Chiang, C. W. Tan, D. P. Palomar, D. O’Neill, and D. Julian, "Power control by geometric programming," IEEE Trans. Wireless Commun., vol. 6, no. 7, pp. 2640-2651, July 2007.

[13] G. L. Stüber, Principles of Mobile Communication, 2nd edition. Kluwer Academic Publishers, 2001.

[14] C. Oestges, N. Czink, B. Bandemer, P. Castiglione, F. Kaltenberger, and A. Paulraj, "Experimental characterization and modeling of outdoorto-indoor and indoor-to-indoor distributed channels," IEEE Trans. Veh. Technol., vol. 59, no. 5, pp. 2253-2265, June 2010.

[15] P. Agrawal and N. Patwari, "Correlated link shadow fading in multi-hop wireless networks," IEEE Trans. Wireless Commun., vol. 8, no. 9, pp. 4024-4036, Aug. 2009.
[16] R. Prasad and A. Kegel, "Improved assessment of interference limits in cellular radio performance," IEEE Trans. Veh. Technol., vol. 40, no. 2, pp. 412-419, May 1991.

[17] Z.-Q. Luo and S. Zhang, "Dynamic spectrum management: complexity and duality," IEEE J. Sel. Topics Signal Process., vol. 2, no. 1, pp. 57-73, Feb. 2008.

[18] M. Pischella and J.-C. Belfiore, "Power control in distributed cooperative OFDMA cellular networks," IEEE Trans. Wireless Commun., vol. 7, no. 3, pp. 1-7, Mar. 2008.

[19] M.-J. Ho and G. L. Stüber, "Co-channel interference of microcellular systems on shadowed Nakagami fading channels," in Proc. IEEE Veh. Technol. Conf., May 1993, pp. 568-571.

[20] X. Hong, C.-X. Wang, and J. Thompson, "Interference modeling of cognitive radio networks," in Proc. IEEE Veh. Tech. Conf., May 2008, pp. 1851-1855.

[21] S. Schwartz and Y. Yeh, "On the distribution function and moments of power sums with lognormal components," Bell Syst. Tech. J., vol. 61, no. 7, pp. 1441-1462, Sep. 1982.

[22] D. C. Schleher, "Generalized Gram-Charlier series with application to the sum of log-normal variates," IEEE Trans. Inf. Theory, vol. 23, no. 2, pp. 275-280, Mar. 1977.

[23] A. A. Abu-Dayya and N. C. Beaulieu, "Outage probabilities in the presence of correlated lognormal interferers," IEEE Trans. Veh. Technol., vol. 43, no. 1, pp. 164-173, Feb. 1994.

[24] N. B. Mehta, J. Wu, A. F. Molisch, and J. Zhang, "Approximating a sum of random variables with a lognormal," IEEE Trans. Wireless Commun., vol. 6, no. 7, pp. 2690-2699, July 2007.

[25] S. Kandukuri and S. Boyd, "Optimal power control in interferencelimited fading wireless channels with outage-probability specifications," IEEE Trans. Wireless Commun., vol. 1, no. 1, pp. 46-55, Jan. 2002.

[26] E. Matskani, N. Sidiropoulos, Z.-Q. Luo, and L. Tassiulas, "Convex approximation techniques for joint multiuser downlink beamforming and admission control," IEEE Trans. Wireless Commun., vol. 7, no. 7, pp. 2682-2693, July 2008.

[27] A. Nasif and B. Mark, "Opportunistic spectrum sharing with multiple cochannel primary transmitters," IEEE Trans. Commun., vol. 8, no. 11, pp. 5702-5710, May 2009.

[28] A. Ben-Tal and A. Nemirovski, "Robust convex optimization," Mathematics Operations Research, vol. 23, no. 24, 1998.

[29] S. Boyd, S.-J. Kim, L. Vandenberghe, and A. Hassibi, "A tutorial on geometric programming," Optim. Eng., vol. 8, pp. 67-127, 2007.

[30] B. R. Marks and G. P. Wright, "A general inner approximation algorithm for nonconvex mathematical programs," Operation Research, vol. 26, no. 4, pp. 681-683, July-Aug. 1978.

[31] E. Dall'Anese, S.-J. Kim, and G. Giannakis, "Admission and power control power allocation for cognitive radio networks by sequential geometric programming," in Proc. Int.'l Conf. Dig. Signal Process., July 2011.

[32] M. Avriel and A. C. Williams, "Complementary geometric programming," SIAM J. Appl. Mathematics, vol. 19, no. 1, pp. 125-141, July 1970.

[33] X. Kang, Y. C. Liang, A. Nallanathan, H. K. Garg, and R. Zhang, "Optimal power allocation for fading channels in cognitive radio networks: ergodic capacity and outage capacity," IEEE Trans. Wireless Commun., vol. 8, no. 2, pp. 940-950, Feb. 2009.

[34] R. Zhang, "On peak versus average interference power constraints for protecting primary users in cognitive radio networks," IEEE Trans. Wireless Commun., vol. 8, no. 4, pp. 2112-2120, Apr. 2009.

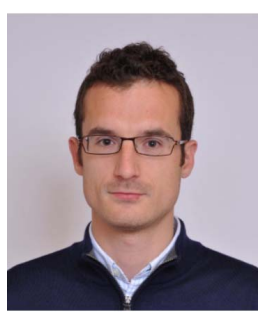

Emiliano Dall'Anese (S'08, M'11) received the Laurea Triennale (B.Sc degree) and the Laurea Specialistica (M.Sc degree) in Telecommunications Engineering from the University of Padova, Italy, in 2005 and 2007, respectively, and the Ph.D in Information Engineering at the Department of Information Engineering (DEI), University of Padova, Italy, in 2011. From January 2009 to September 2010 he was a visiting scholar at the Department of Electrical and Computer Engineering, University of Minnesota, USA. He is currently a post-doctoral associate at the Department of Electrical and Computer Engineering, University of Minnesota, USA.

His research interests lie in the areas of statistical signal processing, communication theory, and networking. Current research focuses on wireless cognitive radio systems, cross-layer design, distributed signal processing, and power grid networks. 


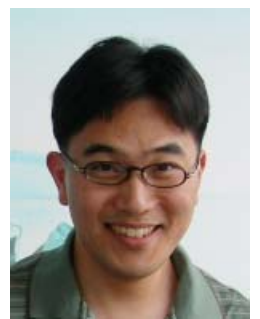

Seung-Jun Kim (M'07) received his B.S. and M.S. degrees from Seoul National University in Seoul, Korea in 1996 and 1998, respectively, and his Ph.D. from the University of California at Santa Barbara in 2005, all in electrical engineering. From 2005 to 2008, he worked for NEC Laboratories America in Princeton, New Jersey, as a research staff member. Since 2008, he has been with the Department of Electrical and Computer Engineering at the University of Minnesota, where he is currently a research assistant professor. His research interests lie in applying signal processing and optimization techniques to various domains including wireless communication and networking, smart power grids, bio and social networks.

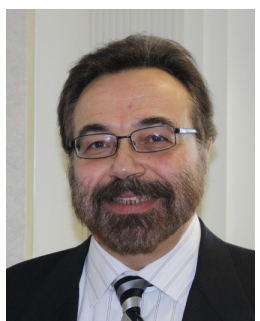

Georgios B. Giannakis (F'97) received his Diploma in Electrical Engr. from the Ntl. Tech. Univ. of Athens, Greece, 1981. From 1982 to 1986 he was with the Univ. of Southern California (USC), where he received his MSc. in Electrical Engineering, 1983, MSc. in Mathematics, 1986, and Ph.D. in Electrical Engr., 1986. Since 1999 he has been a professor with the Univ. of Minnesota, where he now holds an ADC Chair in Wireless Telecommunications in the ECE Department, and serves as director of the Digital Technology Center.

His general interests span the areas of communications, networking and statistical signal processing - subjects on which he has published more than 300 journal papers, 500 conference papers, 20 book chapters, two edited books and two research monographs. Current research focuses on compressive sensing, cognitive radios, network coding, cross-layer designs, wireless sensors, social and power grid networks. He is the (co-) inventor of twenty patents issued, and the (co-) recipient of seven paper awards from the IEEE Signal Processing (SP) and Communications Societies, including the G. Marconi Prize Paper Award in Wireless Communications. He also received Technical Achievement Awards from the SP Society (2000), from EURASIP (2005), a Young Faculty Teaching Award, and the G. W. Taylor Award for Distinguished Research from the University of Minnesota. He is a Fellow of EURASIP, and has served the IEEE in a number of posts, including that of a Distinguished Lecturer for the IEEE-SP Society.

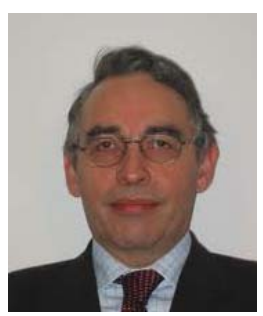

Silvano Pupolin (S'68, M'71, SM'83) received the Laurea degree in Electronic Engineering from the University of Padova, Italy, in 1970. Since then he joined the Department of Information Engineering, University of Padova, where currently is Full Professor of Electrical Communications. He was Chairman of the Faculty of Electronic Engineering from 1990 to 1994, Chairman of the PhD Course in Electronics and Telecommunications Engineering from 1991 to 1997 and Director of the PhD School in Information Engineering from 2004 to 2007. Chairman of the board of PhD School Directors of the University of Padova from 2005 to 2007. He was member of the programming and development committee from 1997 to 2002 and member of Scientific Committee from 1996 to 2001 of the University of Padova; member of the budget Committee of the Faculty of Engineering from 2003 to 2009. Director of CNIT from 2008 to 2010. He has been actively engaged in research on: Digital communication systems over copper wires and fiber optics; Spread spectrum communication systems; Design of large reliable communications networks; Effects of phase noise and HPA nonlinearities in OFDM systems; 3G mobile radio communications systems (UTRA-FDD e TDD) and beyond 3G (OFDM modulation and MCCDMA); Packet radio, Ad-hoc networks with the use of Bluetooth and WLAN. 